\title{
The need to apply the best therapy in heart failure - the era after PARADIGM-HF
}

Malgorzata Lelonek

Department of Noninvasive Cardiology, Medical University of Lodz, Lodz, Poland

Submitted: 28 February 2016

Accepted: 18 April 2016

Arch Med Sci 2017; 13, 5: 1244-1248

DOI: $10.5114 /$ aoms.2016.59711

Copyright @ 2016 Termedia \& Banach

About 26 million adults worldwide suffer from heart failure (HF) [1]. However, general public awareness of HF is poor [2]. Despite the improvement in health care over the past 20 years, mortality in patients with HF remains unacceptably high. About $2-17 \%$ of patients admitted to hospital die during hospitalization, $17-45 \%$ within 1 year of admission and the majority by 5 years from admission [3]. Despite the use of angiotensin-converting enzyme (ACE) inhibitors, $\beta$-blockers, and mineralocorticoid receptor antagonists (MRA), in HF the endogenous neurohormonal system plays an important role.

The molecular complex of LCZ696, a first-in-class angiotensin receptor neprilysin inhibitor (ARNI), combines an angiotensin receptor blocker (valsartan) with a neprilysin inhibitor (sacubitril), and represents an important step in the management of HF and reduced ejection fraction. This dual action places this drug at the center of two critically important systems in HF: the renin-angiotensin-aldosterone system (RAAS), and the natriuretic peptide system (NPS). The mechanism of action for valsartan is well known and for sacubitril is prevention of the catabolism of natriuretic peptides (NPs). Neprilysin (NEP), a neutral endopeptidase, diminishes vasorelaxant, natriuretic, and diuretic actions of NPs beneficial in HF.

Natriuretic peptides exert their effects through binding to their receptors and resulting in the generation of cyclic guanosine monophosphate (cGMP). Cyclic guanosine monophosphate mediates natriuresis and inhibition of renin and aldosterone, and induces vasorelaxant, antifibrotic and antihypertrophic effects [4]. The NPs have a short-lived action due to their rapid metabolism by NEP. Therefore, the use of a substance that blocks the action of NEP, such as a NEP inhibitor (sacubitril), will extend their life, increase their blood levels and consequently increase their effectiveness in the treatment of HF [4].

In the recent Prospective comparison of Angiotensin Receptor neprilysin inhibitor with Angiotensin converting enzyme inhibitors to Determine Impact on Global Mortality and morbidity in Heart Failure trial (PARADIGM-HF), chronic administration of sacubitril/valsartan (formerly known as LCZ696) was superior to enalapril in reducing death and hospitalizations in patients with chronic HF and a reduced ejection fraction (HFrEF) [5]. The dose of enalapril was selected based on its effect in reducing the risk of death in the SOLVD-T (Studies of Left Ventricular Dysfunction (SOLVD) Treatment Trial). Sacubitril/valsartan at a target dose of 97/103 mg (200 mg of LCZ696) twice daily reduced cardiovascular mortality by $20 \%$ compared to an evidence-based dose of the ACE inhibi-

\author{
Corresponding author: \\ Prof. Malgorzata Lelonek \\ $\mathrm{MD}, \mathrm{PhD}$ \\ Department of Noninvasive \\ Cardiology \\ Medical University of Lodz \\ 113 Zeromski St \\ 90-549 Lodz, Poland \\ Phone: +48422014310 \\ E-mail: mlelonek@poczta.fm
}


tor enalapril (10 g twice daily), and death from any cause by $16 \%$ [5]. The magnitude of the beneficial effects of sacubitril/valsartan, as compared to enalapril, was as great as seen with enalapril compared to placebo but was obtained when sacubitril/valsartan was added to background treatment with a $\beta$-blocker and a mineralocorticoid receptor antagonist. The benefit of LCZ696 was consistent across the spectrum of risk, and those at high risk of adverse outcomes obtained a particularly large absolute benefit from sacubitril/valsartan compared with enalapril [6].

The baseline demographics of patients in PARADIGM-HF $[5,7]$ are similar to those of the population of SOLVD-T. Although the majority of patients in PARADIGM-HF were in NYHA functional class II or III at the time of randomization, the median NT-proBNP concentration was high (Table I). In PARADIGM-HF [5], patients without a history of HF-hospitalization within 12 months were required to have either BNP $\geq 150 \mathrm{pg} / \mathrm{ml}$ or NT-proBNP of $\geq 600 \mathrm{pg} / \mathrm{ml}$ in order to be enrolled. Patients with lower levels of natriuretic peptides (BNP $\geq 100 \mathrm{pg} / \mathrm{ml}$ or NT-proBNP $\geq 400 \mathrm{pg} / \mathrm{ml}$ ) were eligible if they had been hospitalized for HF within 12 months. Patients enrolled had an ejection fraction of $\leq 40 \%$ (changed to $\leq 35 \%$ by amendment). Patients taking any dose of ACE inhibitors or angiotensin receptor blockers (ARBs) were considered for enrollment, but were required to tolerate

Table I. Baseline characteristics of patients in PARADIGM-HF [5, 7]

\begin{tabular}{|c|c|c|}
\hline Parameter & $\begin{array}{c}\text { LCZ696 } \\
N=4187\end{array}$ & $\begin{array}{l}\text { Enalapril } \\
N=4212\end{array}$ \\
\hline Age [years] & $63.8 \pm 11.5$ & $63.8 \pm 11.3$ \\
\hline Female gender, $n$ (\%) & $879(21.0)$ & $953(22.6)$ \\
\hline Systolic blood pressure [mm Hg] & $122 \pm 15$ & $121 \pm 15$ \\
\hline Heart rate [beats/min] & $72 \pm 12$ & $73 \pm 12$ \\
\hline Body-mass index & $28.1 \pm 5.5$ & $28.2 \pm 5.5$ \\
\hline Serum creatinine $[\mathrm{mg} / \mathrm{dl}]$ & $1.13 \pm 0.3$ & $1.12 \pm 0.3$ \\
\hline Ischemic cardiomyopathy, $n$ (\%) & $2506(59.9)$ & $2530(60.1)$ \\
\hline Left ventricular ejection fraction (\%) & $29.6 \pm 6.1$ & $29.4 \pm 6.3$ \\
\hline Median B-type natriuretic peptide (IQR) [pg/ml] & $255(155-474)$ & $251(153-465)$ \\
\hline Median N-terminal pro-B-type natriuretic peptide (IQR) $[\mathrm{pg} / \mathrm{ml}]$ & $1631(885-3154)$ & $1594(886-3305)$ \\
\hline NYHA functional class I, $n(\%)$ & $180(4.3)$ & $209(5.0)$ \\
\hline NYHA functional class II, $n(\%)$ & $2998(71.6)$ & $2921(69.3)$ \\
\hline NYHA functional class III, $n$ (\%) & $969(23.1)$ & $1049(24.9)$ \\
\hline NYHA functional class IV, $n(\%)$ & $33(0.8)$ & $27(0.6)$ \\
\hline Hypertension, $n$ (\%) & $2969(70.9)$ & $2971(70.5)$ \\
\hline Diabetes, $n(\%)$ & $1451(34.7)$ & $1456(34.6)$ \\
\hline Atrial fibrillation, $n(\%)$ & $1517(36.2)$ & $1574(37.4)$ \\
\hline Hospitalization for heart failure, $n(\%)$ & $2607(62.3)$ & $2667(63.3)$ \\
\hline Myocardial infarction, $n(\%)$ & $1818(43.4)$ & $1816(43.1)$ \\
\hline Stroke, $n(\%)$ & $355(8.5)$ & $370(8.8)$ \\
\hline$\beta$-Blockers, $n$ (\%) & 3899 (93.1) & 3912 (92.9) \\
\hline MRA, $n(\%)$ & $2271(54.2)$ & $2400(57.0)$ \\
\hline ICD, $n(\%)$ & $623(14.9)$ & $620(14.7)$ \\
\hline CRT, $n(\%)$ & $292(7.0)$ & $282(6.7)$ \\
\hline Digitalis, $n$ (\%) & $1223(29.2)$ & $1316(31.2)$ \\
\hline Diuretics, $n$ (\%) & 3363 (80.3) & 3375 (80.1) \\
\hline
\end{tabular}

MRA - mineralocorticoid receptor antagonist, CRT - cardiac resynchronization therapy, ICD - implantable cardioverter-defibrillator. 
the equivalent of enalapril $10 \mathrm{mg}$ daily for at least 4 weeks before screening along with stable doses of $\beta$-blocker and MRA if indicated. According to the study protocol patients first received enalapril $10 \mathrm{mg}$ twice daily for 2 weeks (single-blind) and then LCZ696 (single-blind) for an additional 4 to 6 weeks, initially at $100 \mathrm{mg}$ twice daily and then $200 \mathrm{mg}$ twice daily. Patients tolerating both drugs at target doses were randomly assigned in a $1: 1$ ratio to double-blind treatment with either enalapril 10 mg twice daily or LCZ696 200 mg twice daily. The trial was stopped early, after a median follow-up of 27 months - an overwhelming benefit with LCZ696.

This beneficial effect on mortality was shown in PARADIGM-HF to a parallel $21 \%$ reduction in cardiac hospitalization for HF, the other component of the primary composite endpoint in PARADIGM-HF. The reduction in heart failure hospitalization with LCZ696 was evident within the first 30 days after randomization. Importantly, the PARADIGM-HF study is one of the few trials demonstrating a reduction in clinical worsening among surviving patients with better health-related quality of life and NYHA class in the sacubitril/valsartan group, compared with the enalapril group [7]. There was also a favorable biomarker response (reductions in NTproBNP and troponin T) in sacubitril/valsartan-treated patients [8].

The $20 \%$ reduction in cardiovascular deaths with LCZ696 relative to enalapril seen during the trial was attributable primarily to reductions in the incidence of both sudden death and death due to progressive HF [9]. Using a putative placebo [10], sacubitril/valsartan showed striking effects on all outcomes examined, with the relative risk reduction for the primary composite endpoint of $39-43 \%$ and cardiovascular mortality $32-34 \%$, and also for all-cause mortality (26-28\% risk reductions) and even larger effects on HF hospitalization, with relative risk reductions of $46-49 \%$.

LCZ696 was better than enalapril in preventing important clinical outcomes and preventing deterioration in symptoms and functional capacity across the broad spectrum of age studied in PARADIGM-HF [11]. Using actuarial estimates from the PARADIGM-HF trial, and assuming that the protective effects of sacubitril/valsartan remain consistent with long-term use, it has been calculated that treatment with sacubitril/valsartan may result in an additional 1 to 2 years life expectancy (and even greater survival free from HF hospitalization) in patients such as those enrolled in the PARADIGM-HF trial: with chronic HF, NYHA class II-IV symptoms, an elevated plasma BNP or NT-proBNP level, and an LVEF of $\leq 40 \%$ [12].

The PARADIGM-HF results suggest that the absolute benefits obtained by switching 1000 patients from an ACE inhibitor/ARB to sacubitril/valsartan over a median treatment period of 27 months would be avoidance of: 47 primary endpoints, 31 cardiovascular deaths, 28 patients hospitalized for HF, 37 patients hospitalized for any reason, 53 admissions for HF and 111 admissions for any reason $[4,8]$.

Overall, fewer patients in the sacubitril/valsartan group than in the enalapril group had a study drug-related adverse event (particularly elevated serum creatinine, elevated serum potassium or cough) and stopped their study medication [4]. However, after randomization, more patients in the sacubitril/valsartan group had symptomatic hypotension than in the enalapril group, but there was no increase in the rate of discontinuation of the study drug for this adverse event. Importantly, intolerance of sacubitril/valsartan leading to treatment withdrawal did not vary greatly according to age and there was no increased risk of serious angioedema.

Sacubitril/valsartan constitutes the first of a new class of drugs, angiotensin receptor neprilysin inhibitors (ARNIs), designed to replace ACE inhibitors and ARBs in HF.

It is important to explain the NT-proBNP and BNP changes with sacubitril/valsartan. The BNP but not NT-proBNP is a substrate for neprilysin [13]. Levels of BNP will reflect the action of the drug, whereas levels of NT-proBNP reflect the effects of the drug on the heart (e.g. on left ventricular filling pressure and wall stress). Levels of plasma BNP were higher during the treatment with sacubitril/valsartan than with enalapril [6]. In contrast, in comparison with enalapril, patients receiving sacubitril/valsartan had consistently lower levels of NT-proBNP, reflecting reduced cardiac wall stress. LCZ696 led to an early (within 4 weeks) and sustained at 8 months $(p<0.0001)$ reduction in NT-proBNP [8], and the clinical findings are supported by the effects on NT-proBNP measured in surviving patients in PARADIGM-HF. The contrasting effects of sacubitril/valsartan on the 2 types of natriuretic peptides (NT-proBNP and $\mathrm{BNP}$ ) represent an important finding, because the levels of the 2 peptides are characteristically parallel with each other during the course of HF. So in the era after PARADIGM-HF, the biomarker for monitoring progress and assessing prognosis in HF should be NT-proBNP, not BNP.

There is more news about related biomarkers in HF. Bayés-Genis et al. identified a positive association between levels of circulating soluble NEP in HF patients and cardiovascular mortality and morbidity, supporting the importance of NEP inhibition as a therapeutic target [14]. Recently they also found that in a multibiomarker strategy in 797 consecutive ambulatory HF patients, only 
Table II. Treatment in heart failure with reduced ejection fraction

\begin{tabular}{|lccc|}
\hline Variable & \multicolumn{2}{c|}{ Mortality in heart failure } & $\begin{array}{c}\text { Hospitalization due to heart } \\
\text { failure }\end{array}$ \\
\cline { 2 - 4 } & Sudden cardiac death & Pump failure & Pump failure \\
\hline$\beta$-Blockers & Reduction & Reduction & Reduction \\
\hline ACEI & Reduction & Reduction & Reduction \\
\hline MRA & Reduction & Reduction & Reduction \\
\hline Ivabradine & Reduction & Reduction & Reduction \\
\hline CRT-D & Reduction & Reduction & Reduction \\
\hline ICD & Reduction & - & - \\
\hline ARNI & Reduction & Reduction & Reduction \\
\hline
\end{tabular}

ACEI - angiotensin-converting enzyme inhibitor, ARNI - angiotensin receptor neprilysin inhibitor, MRA - mineralocorticoid receptor antagonist, CRT-D - cardiac resynchronization therapy with defibrillator function, ICD - implantable cardioverter-defibrillator.

soluble NEP remained an independent prognostic marker of the primary composite endpoint of cardiovascular death or HF hospitalization ( $\mathrm{HR}=$ 1.14; $95 \% \mathrm{Cl}: 1.02-1.27 ; p=0.03)$ and with cardiovascular death $(\mathrm{HR}=1.15 ; 95 \% \mathrm{Cl}: 1.01-1.31$; $p=0.04$ ), while $\mathrm{N}$-terminal pro-B-type natriuretic peptide lost significance in these analyses [15].

The current HFrEF pharmacotherapy is based on low-cost generic medications. We have no real-world estimates of sacubitril/valsartan cost effectiveness. The only publication, by King et al. [16], emphasized the need to make the decision of this intervention based on attempts to determine whether the extra benefit with sacubitril/ valsartan is worth the additional costs. Using a Markov model, the authors revealed that sacubitril/valsartan therapy, compared to enalapril, was more costly $(\$ 60,391$ vs. $\$ 21,758)$ and more effective (6.49 vs. 5.74 quality-adjusted life years (QALYs)) over a lifetime. However, it seems the low-cost generic status of angiotensin-converting enzyme inhibitors (ACEIs) and ARBs might be more attractive for payers than the reduction of $\mathrm{HF}$ hospitalizations observed in PARADIGM-HF. King et al. also reported the relationship between the cost-effectiveness of sacubitril/valsartan and duration of treatment: from $\$ 249,411$ per QALY at 3 years to $\$ 50,959$ per QALY gained over a lifetime. Finally, as with all new therapies, real-world estimates of sacubitril/valsartan cost effectiveness are needed.

In conclusion, PARADIGM-HF is the first successful active controlled trial with a proven comparator (enalapril) in $\mathrm{HF}$, the largest trial in chronic $\mathrm{HF}$ and with the first drug that has proved to be superior to enalapril. The ARNI sacubitril/valsartan combination doubles the effect on cardiovascular death of current inhibitors of the renin-angiotensin system. Therefore, this trial will mark the end of the era of ACE inhibitors/ARBs in HFrEF. The time has come to re-define optimal medical treat- ment in patients with HFrEF and to update the ESC guidelines published in 2012. Table II summarizes the approach in management in HFrEF to reduce mortality and hospitalization due to HF.

\section{Acknowledgments}

I thank Prof. John McMurray (Glasgow) for expert remarks.

\section{Conflict of interest}

The author is an investigator in the PARAGONHF trial.

\section{References}

1. Biu Al. Horwich TB, Fonarow GC. Epidemiology and risk profile of heart failure. Nat Rev Cardiol 2011; 8: 30-41.

2. Lainscak M, Letonja M, Kovacic D, et al. General public awareness of heart failure: results of questionnaire survey during Heart Failure Awareness Day 2011. Arch Med Sci 2014; 10: 355-60.

3. Ponikowski P, Anker SD, Alhabib KF, et al. Heart failure: preventing disease and heath worldwide. ESC Heart Failure 2014; 1: 4-25.

4. Chrysant SG. LCZ-696. Drugs Future 2011; 36: 183-90.

5. McMurray JJ, Packer M, Desai AS, et al. Angiotensinneprilysin inhibition versus enalapril in heart failure. N Engl J Med 2014; 371: 993-1004.

6. Simpson J, Jhund PS, Silva Cardoso J, et al. Comparing LCZ696 with enalapril according to baseline risk using the MAGGIC and EMPHASIS-HF Risk Scores: an analysis of mortality and morbidity in PARADIGM-HF. J Am Coll Cardiol 2015; 66: 2059-71.

7. McMurray JJ, Packer M, Desai AS, et al. Baseline characteristics and treatment of patients in prospective comparison of ARNI with ACEI to Determine Impact on Global Mortality and morbidity in Heart Failure trial (PARADIGM-HF). Eur J Heart Fail 2014; 16: 817-25.

8. Packer M, McMurray J, Desai AS, et al. Angiotensin receptor neprilysin inhibition compared with enalapril on the risk of clinical progression in surviving patients with heart failure. Circulation 2015; 131: 54-61.

9. Desai AS, McMurray J, Packer M, et al. Effect of the angiotensin-receptor-neprilysin inhibitor LCZ696 com- 
pared with enalapril on mode of death in heart failure patients. Eur Heart J 2015; 36: 1990-7.

10. McMurray J, Packer M, Desai AS, et al. A putative placebo analysis of the effects of LCZ696 on clinical outcomes in heart failure. Eur Heart J 2015; 36: 434-9.

11. Jhund PS, Fu M, Bayram E, et al. Efficacy and safety of LCZ696 (sacubitril-valsartan) according to age: insights from PARADIGM-HF. Eur Heart J 2015; 36: 2576-84.

12. Claggett B, Packer M, McMurray J, et al. Estimating the long-term treatment benefits of sacubitril-valsartan. N Engl J Med 2015; 373: 2289-90.

13. Solomon SD, Zile M, Pieske B, et al. Prospective comparison of ARNI with ARB on management of heart failure with preserved ejection fraction (PARAMOUNT) Investigators. The angiotensin receptor neprilysin inhibitor LCZ696 in heart failure with preserved ejection fraction: a phase 2 double-blind randomised controlled trial. Lancet 2012; 380: 1387-95.

14. Bayés-Genís A, Barallat J, Galán A, et al. Soluble neprilysin is predictive of cardiovascular death and heart failure hospitalization in heart failure patients. J Am Coll Cardiol 2015; 65: 657-65.

15. Bayes-Genis A, Barallat J, Galán A, et al. Multimarker strategy for heart failure prognostication. Value of neurohormonal biomarkers: neprilysin vs. NT-proBNP. Rev Esp Cardiol (Engl Ed) 2015; 68: 1075-84.

16. King JB, Shah RU, Bress AP, Nelson RE, Bellows BK. Cost-effectiveness of sacubitril-valsartan combination therapy compared with enalapril for the treatment of heart failure with reduced ejection fraction. JACC Heart Fail 2016; 4: 392-402. 